\title{
Wave fields and nearshore currents in the coastal region opposite San Mauro Cilento (Italy)
}

\author{
FEDERICA PALLESCHI, BENEDETTA IELE, MARCO TAMBURRINO \\ Department of Civil, Constructional and Environmental Engineering \\ "Sapienza" University of Rome \\ Via Eudossiana 18, 00184 \\ ITALY
}

\begin{abstract}
In this paper in order to simulate nearshore currents in computational domains representing the complex morphology of real coastal regions we use a model based on a contravariant integral form of the fully nonlinear Boussinesq equations (FNBE). The contravariant integral form, in which Christoffel symbols are absent, of the continuity equation does not contain the dispersive term. The Boussinesq equation system is numerically solved by a hybrid finite volume-finite difference scheme. The wave breaking is represented by discontinuities of the weak solution of the integral form of the nonlinear shallow water equations (NSWE). The capacity of the proposed model to correctly simulate the wave train propagation on a highly distorted grid is verified against test case present in the literature. The simulation of wave fields and nearshore currents in the coastal region, opposite San Mauro Cilento (Italy) in presence of a system of T-head groins, is numerically reproduced by using the proposed model.
\end{abstract}

Key-Words: - Fully Nonlinear Boussinesq Equations, integral contravariant formulation, dispersive terms, highly distorted grid, San Mauro Cilento, nearshore currents

Received: September 6, 2019. Revised: February 17, 2020. Accepted: April 14, 2020. Published: April 30, 2020.

\section{Introduction}

The model based on the two-dimensional Boussinesq equations is used for the simulation of hydrodynamic phenomena (surface wave transformation, wave breaking, shoreline movement and nearshore currents) which occur in coastal regions because the three-dimensional simulation of the velocity fields [1-3] requires considerable computational time for the long-term sea bottom simulations. The coastal region is characterized by complex morphology, in which slightly sloping and regular sea beds alternate with steep irregular bottoms, and by the presence of anthropic structures and/or river mouths. In order to simulate hydrodynamic phenomena over computational domains characterized by a complex boundary, two strategies can be followed: the first one is represented by the possibility of using unstructured grids; the second one is based on the numerical integration of the governing equations on a generalized curvilinear boundary conforming grid in which the motion equations can be written in contravariant formulation.

In recent literature the Boussinesq equations (integral forms or differential conservative forms) are expressed in terms of conserved variables, in which convective terms are expressed in divergence form and the term related to total local depth is expressed in a gradient form. The simulation of wave propagation from deep water regions up to the coastline is conducted by solve the integral forms or of the differential conservative forms of Boussinesq equations (which in the surf zone reduce to the NSWE by switching off dispersive terms). Breaking waves can be represented by discontinuities of the weak solution of the integral form of the nonlinear shallow water equations (NSWE), numerically solved by a shock-capturing scheme [4]. The Boussinesq equations, in integral or differential conservative forms, do not need any additional term to take into account the wave breaking energy dissipation and do not require any empirical calibration. Roeber et al. [5] adopted a strategy consisting in using a hybrid finite volume-finite difference scheme in order to apply a shockcapturing method to Boussinesq equations expressed in differential conservative form. In this paper a model based on an integral form of the Fully Nonlinear Boussinesq Equations in contravariant formulation, in which terms up to $O\left(\varepsilon^{2} \mu^{2}\right)$ (with $\varepsilon=a_{0} / h_{0}$ and $\mu=h_{0} / L_{0}$ in which $a_{0}$ is the wave amplitude) and second order vertical vorticity terms are included, is presented. In this contravariant integral form, the continuity equation does not contain any dispersive term. The proposed Fully Nonlinear Boussinesq Equations in a contravariant integral form are devoid by the Christoffel symbols and are numerically solved by a hybrid finite 
volume-finite difference scheme. The convective terms and the terms related to the gradient of the square of the total local water depth are discretized by a high-order upwind WENO shock-capturing finite volume scheme based on an exact Riemann solver [6]. A cell centred finite difference scheme are used in order to discretize the dispersive terms and the terms related to the approximation to the second order of the vertical vorticity. No additional term in equations is used in order to take into account the wave breaking energy because the wave breaking is represented by discontinuities of the weak solution of the integral form of the NSWE.

A test case is numerically reproduced in order to verify the capacity of the model to correctly simulate the wave train propagation on a highly distorted grid. The proposed model is also used for the application on a real engineering case regarding the simulation of wave fields and nearshore currents in the coastal region opposite San Mauro Cilento (Italy).

\section{Governing Equations}

Let $H=h+\eta$ be the total local water depth, where $h$ is the local still water depth and $\eta$ is free surface elevation with respect to the undisturbed free surface. The vertical distribution of the horizontal velocity $\vec{U}(z)$, if we consider a Taylor expansion of the velocity about an arbitrary distance from the still water surface, $\sigma$, and assume zero horizontal vorticity, can be written as

$$
\vec{U}(z)=\vec{u}+\vec{v}(z)
$$

where $\vec{u}$ is the horizontal velocity at an arbitrary distance from the still water level $z=\sigma$.

$$
\begin{gathered}
\vec{v}(z)=(\sigma-z) \nabla(\nabla \cdot(h \vec{u}))+ \\
\left(\frac{\sigma^{2}}{2}-\frac{\mathrm{z}^{2}}{2}\right) \nabla(\nabla \cdot(\vec{u}))
\end{gathered}
$$

Equation (2) represent the second order term in power series expansion of the velocity vector about $\sigma$, in which $\nabla$ is the two-dimensional differential operator defined as $\nabla=\left(\frac{\partial}{\partial x}, \frac{\partial}{\partial y}\right)$ in a Cartesian reference system.

The depth averaged value of $\vec{v}(z)$, obtained by retaining terms of order $O\left(\mu^{2}, \varepsilon^{2} \mu^{2}\right)$, is defined $\overrightarrow{\vec{v}}$ and is written as

$$
\overrightarrow{\vec{v}}=\frac{1}{H} \int_{-h}^{\eta} \vec{v}(z) d z=
$$

$$
\begin{gathered}
\left(\frac{\sigma^{2}}{2}-\frac{1}{6}\left(h^{2}-h \eta+\eta^{2}\right)\right) \nabla(\nabla \cdot(\vec{u})) \\
+\left(\sigma+\frac{1}{2}(h-\eta)\right) \nabla(\nabla \cdot(h \vec{u}))
\end{gathered}
$$

The transformation from the Cartesian coordinate system $\vec{x}$ to the curvilinear coordinate system $\vec{\xi}$ (henceforth the superscript indicates components and not powers) is defined by $x^{l}=$ $x^{l}\left(\xi^{1}, \xi^{2}\right)$ (with $\left.l=1,2\right)$. Let $\vec{g}_{(l)}=\partial \vec{x} / \partial \xi^{l}$ and $\vec{g}^{(l)}=\partial \xi^{l} / \partial \vec{x}$ be respectively the covariant and contravariant base vector. The metric tensor and its inverse are given respectively by $g_{l m}=\vec{g}_{(l)} \cdot \vec{g}_{(m)}$ and $g^{l m}=\vec{g}^{(l)} \cdot \vec{g}^{(m)}$. The Jacobian of the transformation is $\sqrt{g}=\sqrt{\operatorname{det}\left(g_{l m}\right)}$. The transformation relationships between the components of the generic vector $\vec{b}$ in the Cartesian coordinate system and its contravariant and covariant components, $b^{l}$ and $b_{l}$, in the curvilinear coordinate system are given in the Appendix.

In order to apply a shock-capturing scheme to the Boussinesq type equations, the convective terms must be expressed in conservative form, i.e. in divergence form. In [7] the system evolution variables were the conserved variables $H$ e $H u^{l}$. The choice of these conserved variables implied the presence of a source term in the mass conservation equation (right-hand side of Equation (4) in [6]). In this paper we choose, as conserved variables, the total local depth $H$ and the contravariant quantity

$$
M^{l}=H\left(u^{l}+\bar{v}^{l}\right)
$$

in which $\left(u^{l}+\bar{v}^{l}\right)$ represents the depth averaged horizontal velocity. For this reason and considering that the bottom depth does not vary over time, the contravariant integral form of the continuity equation can be written as

$$
\iint_{\Delta A} \frac{\partial H}{\partial t} d A+\int_{L} M^{m} n_{m} d L=0
$$

where $L$ is the contour line of the surface element of area $\Delta A$ and $n_{m}$ is the $\mathrm{m}$-th component of the covariant outward normal. The continuity equation can be written without any source term, but only with a flux term (second term of Equation 5), due to the choice of the conserved variable $M^{l}$ expressed by Equation (4). This result implies that the continuity equation can be solved entirely by a high order shock-capturing finite volume scheme. 
With this new conserved variable, the integral over the surface element of area $\Delta A$ of the contravariant momentum equation can be written as

$$
\begin{gathered}
\iint_{\Delta A} \frac{\partial M^{1}}{\partial t} d A+\iint_{\Delta A}\left(\frac{M^{l} M^{m}}{H}\right)_{, m} d A \\
=-\iint_{\Delta A} G H g^{l m} \eta_{, m} d A \\
-\iint_{\Delta A} H\left(V^{l}+T^{l}+W^{l}+R^{l}\right) d A \\
-\iint_{\Delta A} H\left(\frac{\partial \bar{v}^{l}}{\partial t}+\bar{v}_{, m}^{l} u^{m}+\right. \\
\left.u_{, m}^{l} \bar{v}^{m}+\bar{v}_{, m}^{l} \bar{v}^{m}\right) d A
\end{gathered}
$$

in which $u^{l}$ and $\bar{v}^{l}$ are the contravariant components of the vectors $\vec{u}$ and $\vec{v} ; G$ is acceleration due to gravity. $R^{l}$ is the contravariant components of the bottom resistance term; $V^{l}$ and $T^{l}$ are the contravariant components of the dispersive terms obtained by retaining terms of order $O\left(\mu^{2}, \varepsilon^{3} \mu^{2}\right)$; $W^{l}$ is the contravariant component of the term related to the second order approximation of the vertical vorticity. $R^{l}, V^{l}, T^{l}$ and $W^{l}$ are expressed by

$$
\begin{gathered}
V^{l}=\frac{\sigma^{\wedge}}{2} g^{l m}\left[\left(\frac{\partial u^{k}}{\partial t}\right)_{, k}\right]_{, m} \\
+\sigma g^{l m}\left[\left(h \frac{\partial u^{k}}{\partial t}\right)_{, k}\right]_{, m} \\
-g^{l m}\left[\frac{1}{2} \eta^{\wedge}\left(\frac{\partial u^{k}}{\partial t}\right)_{, k}+\eta\left(h \frac{\partial u^{k}}{\partial t}\right)_{, k}\right]_{, m} \\
T^{l}=g^{l m}\left\{(\sigma-\eta) u^{i}\left(\left[h u^{k}\right]_{, k}\right)_{, i}\right. \\
\left.+\frac{1}{2}\left(\sigma^{\wedge}-\eta^{\wedge}\right) u^{i}\left(u_{, k}^{k}\right)_{, i}\right\}_{, m} \\
+\frac{1}{2} g^{l m}\left\{\left[\left(h u^{k}\right)_{, k}+\eta u_{, k}^{k}\right]^{\wedge 2}\right\}_{, m} \\
W^{l}=\left(\varepsilon^{m i} g_{i p} u_{, m}^{p}\right) \varepsilon^{j l} \bar{v}_{j} \\
+\left(\varepsilon^{m i} g_{i p} \bar{v}_{, m}^{p}\right) \varepsilon^{j l} u_{j}
\end{gathered}
$$

in which

$$
\varepsilon^{m i}=
$$

$\left\{\begin{array}{c}\frac{1}{\sqrt{g}} \text { if }(m, i) \text { is a even permutation of }(1,2) \\ -\frac{1}{\sqrt{g}} \text { if }(m, i) \text { is a odd permutation of }(1,2) \\ 0 \text { if the indexes are equal }\end{array}\right.$

The "^" symbol indicates the operation of power raising and $\eta_{c}$ is an arbitrary constant value.
The source terms are perfectly balanced by the flux terms in motion equations that admit stationary solutions.

A numerical scheme is said to be well-balanced and satisfies the C-Property if it preserves the steady state solutions exactly. The surface gradient term could be split into a source term that is related to the bed slope and a term related to the gradient of the square of the local total depth, in order to include this term into the flux term and to perform a shockcapturing upwind scheme.

The source term relative to the bottom slope should perfectly balance the numerical discretization of the term related to the gradient of the square of the total local depth, when there is no motion.

Shi et al [8] point out that the above decomposition induces a numerical imbalance problem and does not allow the numerical scheme to satisfy the C-Property for non-uniform bed.

The surface gradient term is decomposed in order to obtain a "well-balanced" numerical scheme as

$$
\begin{gathered}
G g^{l m} H \eta_{, m}=\left(G g^{l m} \frac{H^{\wedge} 2}{2}\right)_{, m} \\
-G\left(\eta-\eta_{c}\right)\left(g^{l m} h\right)_{, m}-G \eta_{c}\left(g^{l m} h\right)_{, m} \\
-G\left(g^{l m} \frac{h^{\wedge} 2}{2}\right)_{, m}
\end{gathered}
$$

We split the term $V^{l}$ on the right-hand side of Equation (6) as follows

$$
V^{l}=\frac{\partial V^{\prime l}}{\partial t}+V^{\prime \prime l}
$$

in which $V^{\prime l}$ and $V^{\prime \prime l}$ are expressed by

$$
\begin{gathered}
V^{\prime l}=\frac{1}{2} \sigma^{\wedge} g^{l m}\left[\left(u^{k}\right)_{, k}\right]_{, m} \\
+\sigma g^{l m}\left[\left(h u^{k}\right)_{, k}\right]_{, m} \\
-g^{l m}\left[\frac{1}{2} \eta^{\wedge}\left(u^{k}\right)_{, k}+\eta\left(h u^{k}\right)_{, k}\right]_{, m} \\
V^{\prime \prime l}=g^{l m}\left[\frac{\partial}{\partial t}\left(\frac{\eta^{\wedge}}{2}\right)\left(u^{k}\right)_{, k}\right]_{, m} \\
+g^{l m}\left[\frac{\partial \eta}{\partial t}\left(h u^{k}\right)_{, k}\right]_{, m}
\end{gathered}
$$

We define with $D^{l}$ an auxiliary variable defined by

$$
D^{l}=H\left(u^{l}+V^{\prime l}\right)
$$


We identify a physical direction with the one of the contravariant base vector $\overrightarrow{\tilde{g}}^{(l)}$ which is defined at the centre of $\Delta \mathrm{A}$, in order to obtain a contravariant integral form of the Fully Nonlinear Boussinesq Equations devoid of Christoffel symbols. We take the projection, in the direction of vector $\overrightarrow{\tilde{g}}^{(l)}$, of the rate of change of the momentum of the material volume of fluid that at the generic instant $t$ coincides with $\Delta \mathrm{A}$. We equate this projection to the projection, in the same direction, of the net force acting on the material volume. By introducing Equations (11), (12) and (15) into Equation (6), and by adopting the above-mentioned procedure we obtain

$$
\begin{aligned}
& \frac{\partial \widetilde{D}^{l}}{\partial t}= \\
& \frac{1}{\Delta A}\left\{-\sum_{\mu=1}^{2}\left[\int _ { \Delta \xi ^ { \mu + } } \left(\overrightarrow{\tilde{g}}^{(l)} \cdot \vec{g}_{(k)} \frac{M^{k} M^{\mu}}{H}+\right.\right.\right. \\
& \left.\overrightarrow{\tilde{g}}^{(l)} \cdot \vec{g}^{(\mu)} G \frac{H^{\wedge} 2}{2}\right) \sqrt{g} d \xi^{v}- \\
& \int_{\Delta \xi^{\mu-}}\left(\overrightarrow{\tilde{g}}^{(l)} \cdot \vec{g}_{(k)} \frac{M^{k} M^{\mu}}{H}+\right. \\
& \left.\left.\overrightarrow{\tilde{g}}^{(l)} \cdot \vec{g}^{(\mu)} G \frac{H^{\wedge} 2}{2}\right) \sqrt{g} d \xi^{\nu}\right]+ \\
& \iint_{\Delta A} G\left(\eta-\eta_{c}\right) \overrightarrow{\tilde{g}}^{(l)} . \\
& \vec{g}_{(k)} g^{k m} h_{, m} \sqrt{g} d \xi^{1} d \xi^{2}+ \\
& G \eta_{c} \sum_{\mu=1}^{2}\left[\int_{\Delta \xi^{\mu+}} \overrightarrow{\tilde{g}}^{(l)} \cdot \vec{g}^{(\mu)} h \sqrt{g} d \xi^{\nu}-\right. \\
& \left.-\int_{\Delta \xi^{\mu-}} \overrightarrow{\tilde{g}}^{(l)} \cdot \vec{g}^{(\mu)} h \sqrt{g} d \xi^{\nu}\right]+ \\
& \frac{G}{2} \sum_{\mu=1}^{2}\left[\int_{\Delta \xi^{\mu+}} \overrightarrow{\tilde{g}}^{(l)} \cdot \vec{g}^{(\mu)} h^{\wedge} \sqrt{g} d \xi^{\nu}-\right. \\
& \left.\int_{\Delta \xi^{\mu-}} \overrightarrow{\tilde{g}}^{(l)} \cdot \vec{g}^{(\mu)} h^{\wedge} \sqrt{g} d \xi^{\nu}\right]- \\
& \iint_{\Delta A} H \overrightarrow{\tilde{g}}^{(l)} \cdot \vec{g}_{(k)} V^{\prime \prime k} \sqrt{g} d \xi^{1} d \xi^{2}- \\
& \iint_{\Delta A} H \overrightarrow{\tilde{g}}^{(l)} \cdot \vec{g}_{(k)}\left(T^{k}+W^{k}+\right. \\
& \left.R^{k}\right) \sqrt{g} d \xi^{1} d \xi^{2}+ \\
& \iint_{\Delta A} \overrightarrow{\tilde{g}}^{(l)} \cdot \vec{g}_{(k)} \frac{\partial H}{\partial t}\left(V^{\prime k}-\bar{v}^{k}\right) \sqrt{g} d \xi^{1} d \xi^{2}+ \\
& \iint_{\Delta A} H\left(\overrightarrow{\tilde{g}}^{(l)} \cdot \vec{g}_{(k)} \bar{v}^{k}\right)_{, m} u^{m} \sqrt{g} d \xi^{1} d \xi^{2}+ \\
& \iint_{\Delta A} H\left(\overrightarrow{\tilde{g}}^{(l)} \cdot \vec{g}_{(k)} u^{k}\right)_{, m} \bar{v}^{m} \sqrt{g} d \xi^{1} d \xi^{2}+ \\
& \left.\iint_{\Delta A} H\left(\overrightarrow{\tilde{g}}^{(l)} \cdot \vec{g}_{(k)} \bar{v}^{k}\right)_{, m} \bar{v}^{m} \sqrt{g} d \xi^{1} d \xi^{2}\right\}
\end{aligned}
$$

where $\widetilde{D}^{l}$ represent the averaged value of $D^{l}$ over the surface element of area $\Delta \mathrm{A}$, defined as

$$
\widetilde{D}^{l}=\frac{1}{\Delta A} \iint_{\Delta A} D^{l} \sqrt{g} d \xi^{1} d \xi^{2}
$$

Over the same element of area $\Delta \mathrm{A}$, the integral form of the continuity Equation (6) reads

$$
\begin{gathered}
\frac{\partial \widetilde{H}}{\partial t}=-\frac{1}{\Delta A} \sum_{\mu=1}^{2}\left[\int_{\Delta \xi^{\mu+}} M^{\mu} \sqrt{g} d \xi^{v}-\right. \\
\left.\int_{\Delta \xi^{\mu-}} M^{\mu} \sqrt{g} d \xi^{v}\right]
\end{gathered}
$$

where $\widetilde{H}$ represents the average value of $H$ over the surface element of area $\Delta \mathrm{A}$

$$
\widetilde{H}=\frac{1}{\Delta A} \iint_{\Delta A} H \sqrt{g} d \xi^{1} d \xi^{2}
$$

Equations (16) and (18) represent a new integral form of the Fully Nonlinear Boussinesq Equations, expressed in a contravariant formulation devoid Christoffel symbols. These equations are accurate to $O\left(\mu^{2}, \varepsilon^{3} \mu^{2}\right)$ in dispersive terms and conserve vertical vorticity with a leading order error of $O\left(\mu^{4}\right)$. In the above-mentioned equations $H$ and $M^{l}$ are the conserved variables. Consequently, the difference between the presented momentum balance equation and the form presented by Cannata et al. [7] consists in the expression of the convective terms.

Furthermore, unlike the [7] model, in the continuity equation no dispersive term is present. This result makes it possible to solve the continuity equation entirely by a high order shock-capturing finite volume scheme. By adopting this model the errors, in the numerical solution, due to the discretization of the dispersive term in the continuity equation, are not introduced. Furthermore, the surface gradient term has been split in order to solve this term by a finite volume technique and to obtain a "well balanced" numerical scheme.

\section{Simulation of wave train propagation on a highly distorted grid} In this section the proposed numerical model based on conservative and contravariant form of the FNBE, is used in order to verify the capacity of the model to simulate the propagation of strongly dispersive and highly nonlinear wave trains $(\varepsilon=$ 0.2 ) on curvilinear highly distorted grid. 


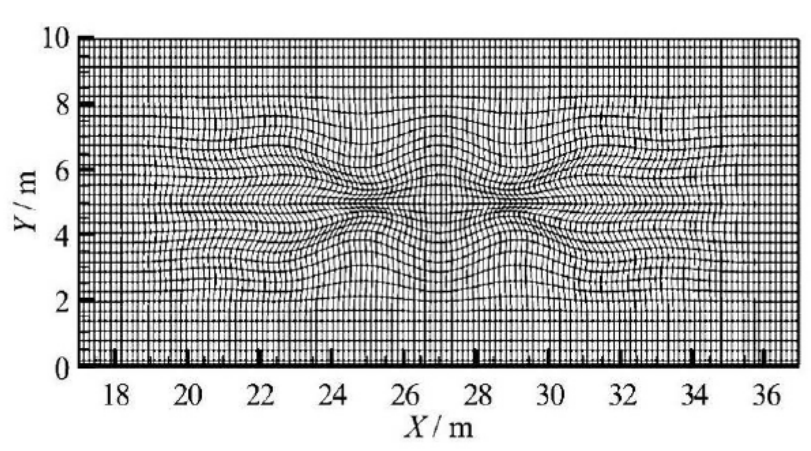

Fig. 1 Simulation of wave train propagation on a highly distorted grid. Grid distortion.

In Figure 1 the calculation grid with the highly distorted zone is shown. In order to correctly dissipate the wave energy, we use two lateral sponge zones. A flat bottom situate at $0.85 \mathrm{~m}$ from the still water level is used to analyse whether the wave profile shows a deformation during the simulation.

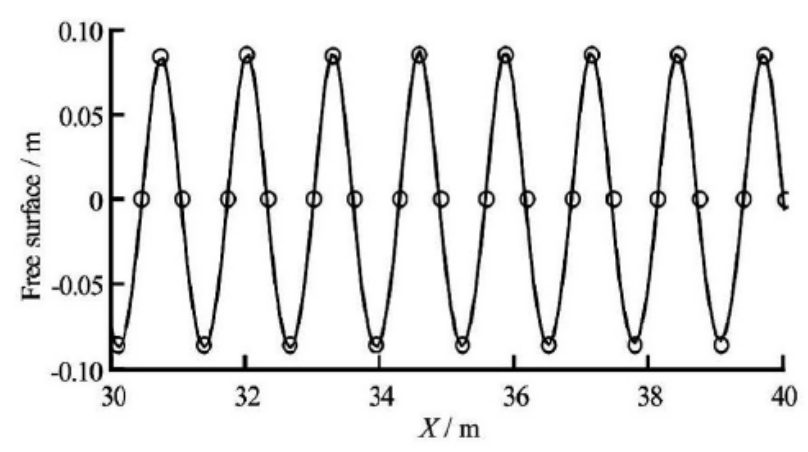

Fig. 2 Wave profile. Wave train: $a=0.17 m, T=$ $0.87 s, \varepsilon=0.2, \mu^{2}=0.5$.

The wave train has an amplitude of $a=0.17 \mathrm{~m}$, a period of $T=0.87 \mathrm{~s}$, a nonlinearity of $\varepsilon=0.2$ and a strong dispersion of $\mu^{2}=0.5$.

In Figure 2 the wave profile after $50 \mathrm{~s}$ of simulation is shown. The high dispersion of the wave train is correctly reproduced by the proposed model. The wave profile does not present any disturbs nor modification of the wave characteristic and it is in good agreement with the data extrapolated from the analytical solution.

The free surface elevation of this wave train is shown in Figure 3. From this figure it is possible to notice that there no any alterations are present, which could have disturbed the wave profile, therefore, the wave form is preserved.

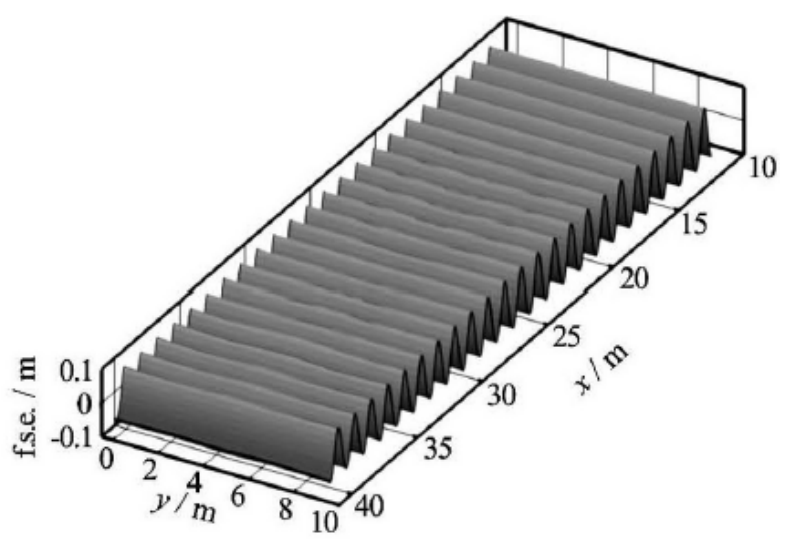

Fig. 3 Instantaneous wave field. Wave train: $a=$ $0.17 m, T=0.87 s, \varepsilon=0.2, \mu^{2}=0.5$.

In conclusion, we demonstrate that the model, based on the contravariant and conservative form of the FNBE (in which the term up to $O\left(\varepsilon^{2} \mu^{2}\right)$ and second order vertical vorticity) and on shockcapturing high order WENO scheme [3], is able to reproduce the propagation of strongly dispersive and highly nonlinear wave trains on curvilinear highly distorted grids.

\section{Simulation of wave field and currents in the coastal region opposite San Mauro Cilento (Italy)}

In this section, the proposed model is applied to the coastal region opposite San Mauro Cilento (Italy). This coastal region is characterized by the presence of several inlets bounded by promontories and rocky protrusions that represent the boundaries of physiographic units. The hydrodynamic fields in a coastal region opposite a portion of coastline, washed by the Tyrrhenian sea, that is bounded by two promontories that delimited a physiographic unit, is studied using the proposed model.

We simulate the wave field and the nearshore currents produced by a wave train with wave heights of $3 \mathrm{~m}$ and a wave period of $10 \mathrm{~s}$ coming from $270^{\circ} \mathrm{N}$. We are interested in the hydrodynamic fields produced by the interaction between the above- mentioned incoming wave train and a system of T-head groins. 


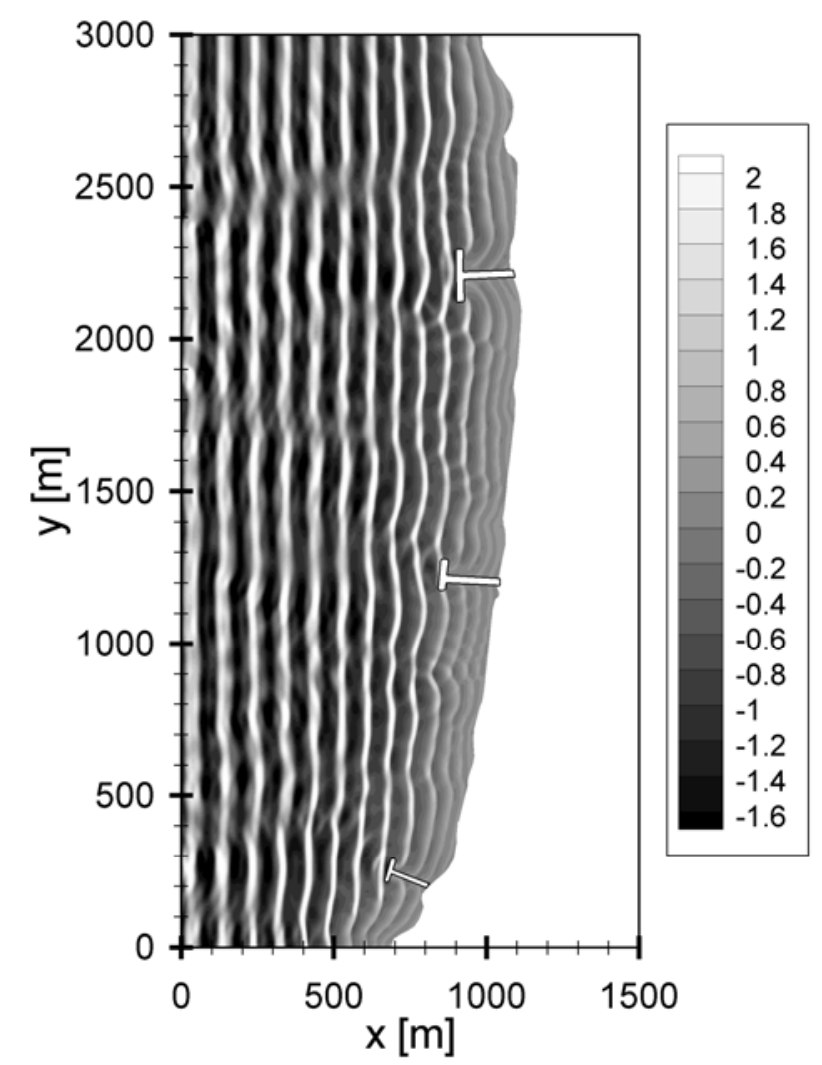

Fig. 4 Simulation of wave field and nearshore currents in the coastal region opposite San Mauro Cilento (Italy). Instantaneous wave field in presence of a system of three T-head groins. Wave train: $H=$ $3 m, T=10 \mathrm{~s}$, direction $270^{\circ} \mathrm{N}$.

In Figure 4, the instantaneous wave field in presence of the T-head groins is shown. Near the coastline, the presence of the T-head groins modifies significantly the wave field: in particular the T-head groins produce a rotation of the wave front and a decree of the wave energy. By doing this, the T-head groins induce the sedimentation of the solid particles that are suspended by the wave breaking and transported towards the T-head groins by the nearshore currents.

In Figure 5, the time averaged velocity field, produced by the wave field represented in Figure 4, is shown. From this figure, it is possible to notice the presence of two nearshore currents produced by the gradient of the averaged wave height: the first one is oriented towards $N-O$, the second nearshore current is opposite to the previous one. The two opposite nearshore currents, at $y=700 \mathrm{~m}$, produce a offshore directed rip current. The T-head groins produce a modification, near the coastline, of the two nearshore currents and induce the eddies, that produce solid particles sedimentation.

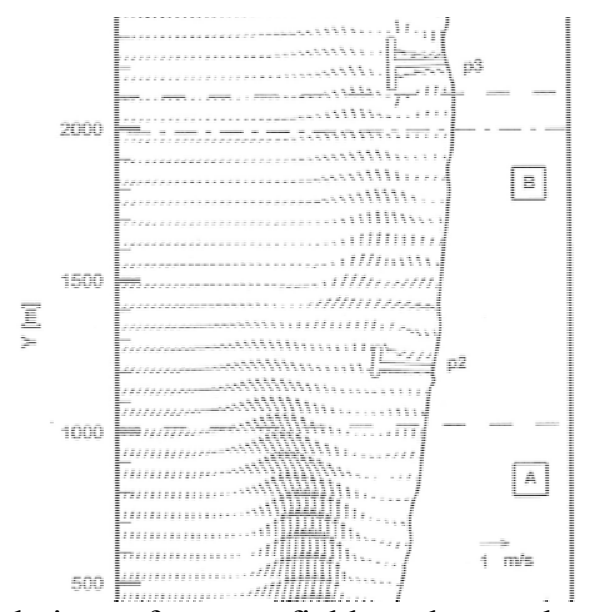

Fig. 5 Simulation of wave field and nearshore currents in the coastal region opposite San Mauro Cilento (Italy). Time averaged velocity field.

Figure 6 represents details of the time averaged velocity field near the T-head groins. In Figure $6 a$, the velocity field in zone $A$ is shown (see Figure 5 for the zone definition). From Figure $6 a$ it is possible to notice that the two nearshore currents that come from opposite directions, produce an offshore directed rip current, at $y=700 \mathrm{~m}$. Near the T-head groin $p 1$, two circulation cells are shown: the first nearshore current is counterclockwise, while the second one is clockwise. These two nearshore currents are able to produce the solid particles sedimentation.

In Figure $6 \mathrm{~b}$, the velocity field in zone $B$ is shown. Downstream of the T-head groin $p 2$, with respect to the nearshore current, a significant reduction of the velocity is shown; this reduction can induce solid particles to sediment near the structure. Upstream of the T-head groin the presence of a circulation cell counterclockwise induces weak sedimentation phenomena.

In Figure $6 \mathrm{c}$, the velocity field in zone $C$ is shown. The presence of the two circulation cells (clockwise upstream of the T-head groin and counterclockwise downstream of the T-head groin) underlines that the structure produces the modification of the main nearshore current and produce solid particles sedimentation. See also [9] and [10].

\section{Conclusion}

In this paper in order to simulate nearshore currents in computational domains representing the complex morphology of real coastal regions we use a model based on a contravariant integral form of the fully nonlinear Boussinesq equations (FNBE).

The presented Fully Nonlinear Boussinesq Equations model has been used for the simulation of 
wave fields and nearshore currents in the coastal region characterized by complex morphology, irregular seabed and by the presence of three T-head groins, in coastal region opposite San Mauro Cilento (Italy).
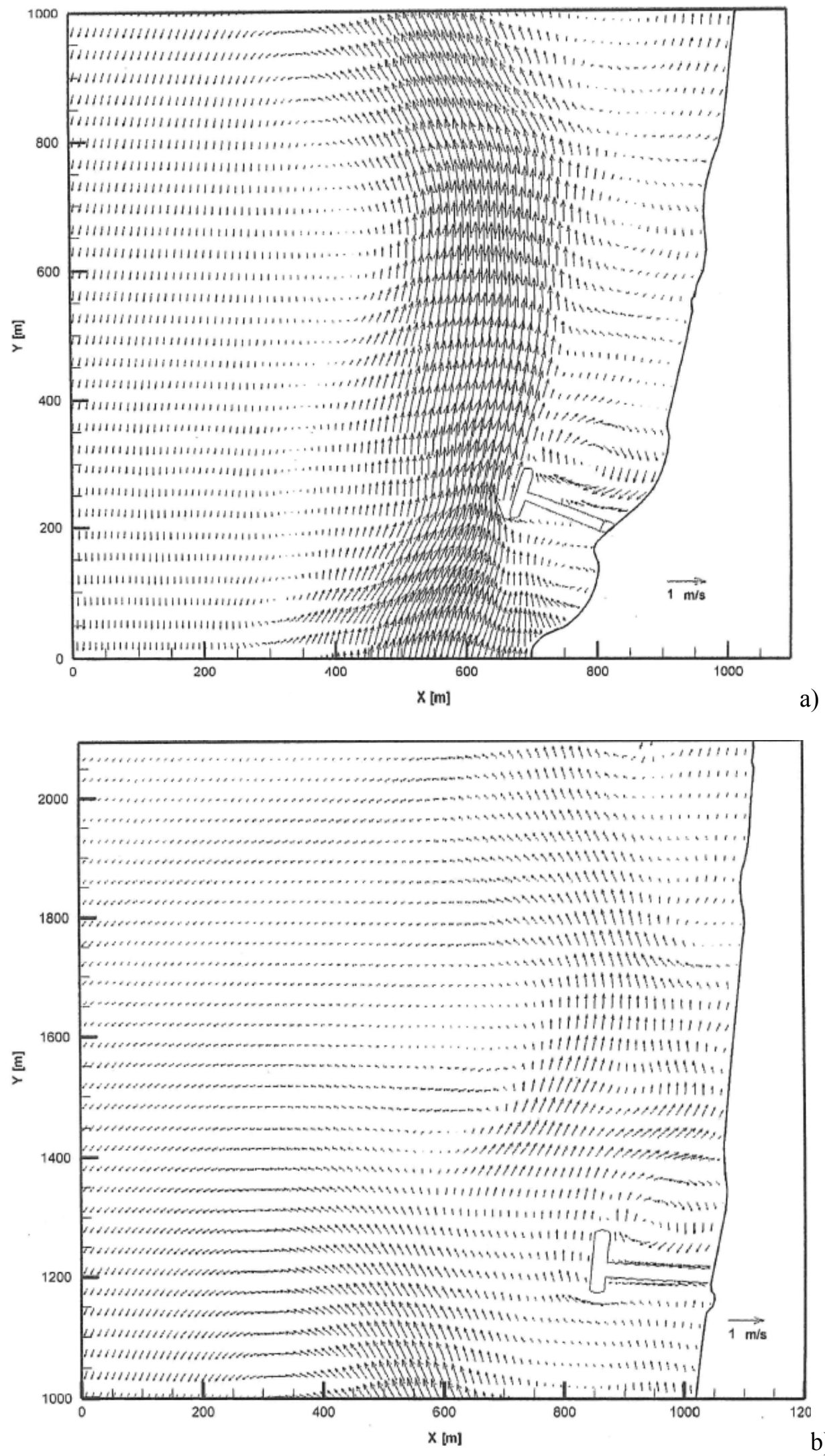


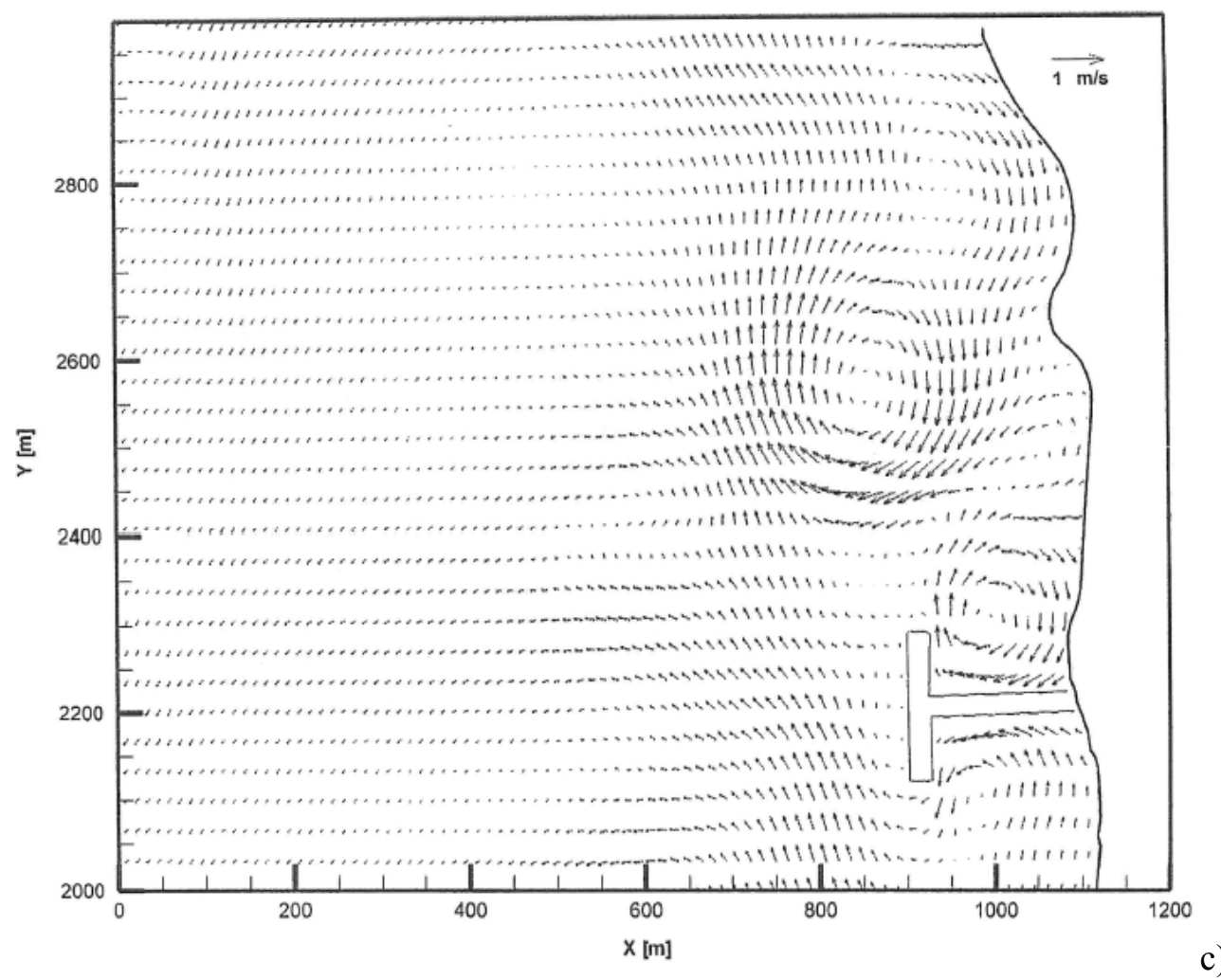

Fig. 6 Simulation of wave field and nearshore currents in the coastal region opposite San Mauro Cilento (Italy). Details of the time averaged velocity field. A vector out of four is presented.

\section{Appendix}

$$
\begin{gathered}
b^{l}=\vec{g}^{(l)} \cdot \vec{b} ; \quad b_{l}=\vec{g}_{(l)} \cdot \vec{b} \\
\vec{b}=b^{l} \vec{g}_{(l)} ; \quad \vec{b}=b_{l} \vec{g}^{(l)} \\
b^{l}{ }_{m}=\partial b^{l} / \partial \xi^{m}+\Gamma_{m k}^{l} b^{k} \\
\Gamma_{m k}^{l}=\vec{g}^{(l)} \cdot \partial \vec{g}_{(k)} / \partial \xi^{m} \\
T_{, m}^{l m}=\frac{1}{\sqrt{g}} \frac{\partial T^{l m} \sqrt{g}}{\partial \xi^{m}}+T^{n m} \Gamma_{n m}^{l}
\end{gathered}
$$

\section{References:}

[1] Cannata G., Gallerano F., Palleschi F., Petrelli C., Barsi L., Three-dimensional numerical simulation of the velocity fields induced by submerged breakwaters, International Journal of Mechanics, Vol. 13, 2019, pp. 1-14.

[2] Cannata G., Petrelli C., Barsi L., Camilli F., Gallerano F. 3D free surface flow simulations based on the integral form of the equations of motion. WSEAS Transaction on Fluid Mechanics, Vol. 12, 2017, pp. 166-175.

[3] Cannata G., Petrelli C., Barsi L., Gallerano F., Numerical integration of the contravariant integral form of the Navier-Stokes equations in time-dependent curvilinear coordinate systems for three-dimensional free surface flows, Continuum Mechanics and Thermodynamics, Vol. 31, No. 2, 2019, pp.491-519.

[4] Cannata G., Petrelli C., Barsi L., Fratello F., Gallerano F., A dam-break flood simulation model in curvilinear coordinates, WSEAS Transactions on Fluid Mechanics, Vol. 13, 2018, pp. 60-70.

[5] Roeber V., Cheung K.F., Kobayashi M.H. Shock-capturing Boussinesq-type model for nearshore wave processes. Coastal Engineering, Vol. 57, No. 4, 2010, pp. 407423.

[6] Gallerano F., Cannata G., Tamburrino M. Upwind WENO scheme for Shallow Water Equations in contravariant formulation. Computers and Fluids, Vol. 62, 2012, pp. 1-12

[7] Cannata G., Barsi L., Petrelli C., Gallerano F., Numerical investigation of wave fields and currents in a coastal engineering case study, WSEAS Transactions on Fluid Mechanics, Vol. 13, 2018, pp. 87-94.

[8] Shi F., Kirby J.T., Harris J.C., Geiman J.D., Grilli S.T., A high-order adaptive time stepping TVD solver for Boussinesq modelling of 
breaking waves and coastal inundation, Ocean Modelling, Vol. 43-44, 2012, pp.35-51.

[9] Cornelia Revnic, Radu Trimbitas, Numerical Simulation of MHD Natural Convection Flow in a Wavy Cavity Filled by a Hybrid $\mathrm{Cu}-\mathrm{AI} 2 \mathrm{O} 3 /$ water Nanofluid with Discrete Heating, WSEAS Transactions on Heat and Mass Transfer, Volume 14, 2019, pp. 158-166

[10] Loganathan Parasuraman, Dhivya Mohanavel, Soret and Dufour effects of Convective Boundary Layer Flow over a Moving Permeable Cylinder, WSEAS Transactions on Heat and Mass Transfer, Volume 14, 2019, pp. 1-12 